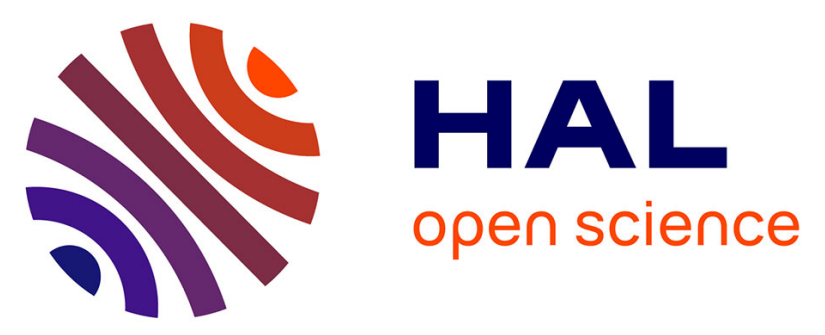

\title{
Mise en évidence par granularité en lumière blanche de phénomènes de multilocalisation de la déformation pour les bétons de poudres réactivessollicités en traction
}

Vincent Huon, Olivier Maisonneuve, Bertrand Wattrisse, Jean-Michel Muracciole

\section{To cite this version:}

Vincent Huon, Olivier Maisonneuve, Bertrand Wattrisse, Jean-Michel Muracciole. Mise en évidence par granularité en lumière blanche de phénomènes de multilocalisation de la déformation pour les bétons de poudres réactivessollicités en traction. Comptes rendus de l'Académie des sciences. Série IIb, Mécanique, 2001, 329, pp.897-900. 10.1016/S1620-7742(01)01416-7 . hal-02457405

\section{HAL Id: hal-02457405 https://hal.science/hal-02457405}

Submitted on 28 Jan 2020

HAL is a multi-disciplinary open access archive for the deposit and dissemination of scientific research documents, whether they are published or not. The documents may come from teaching and research institutions in France or abroad, or from public or private research centers.
L'archive ouverte pluridisciplinaire HAL, est destinée au dépôt et à la diffusion de documents scientifiques de niveau recherche, publiés ou non, émanant des établissements d'enseignement et de recherche français ou étrangers, des laboratoires publics ou privés. 


\title{
Mise en évidence par granularité en lumière blanche de phénomènes de multilocalisation de la déformation pour les bétons de poudres réactives sollicités en traction
}

\author{
Vincent HUON ${ }^{a}$, Olivier MAISONNEUVE ${ }^{\text {b }}$, Bertrand WATTRISSE ${ }^{a}$, \\ Jean Michel MURACCIOLE ${ }^{\text {a }}$ \\ a Laboratoire de mécanique et génie civil, UMR 5508 CNRS-UMII, Université Montpellier II, \\ c.c. 81, place Eugène Bataillon, 34095 Montpellier cedex 05, France \\ ${ }^{b}$ Laboratoire de mécanique et génie civil, UMR 5508 CNRS-UMII, Université Montpellier II, \\ c.c. 48, place Eugène Bataillon, 34095 Montpellier cedex 05, France \\ Courriel : huon@Imgc.univ-montp2.fr; maisonneuve@Imgc.univ-montp2.fr; wattrisse@lmgc.univ-montp2.fr; \\ muracciole@Imgc.univ-montp2.fr
}

Résumé. Le comportement en traction d'un béton de poudres réactives est étudié à partir d'observations cinématiques par granularité en lumière blanche fondées sur une technique d'intercorrélation d'images. Les mesures mettent en évidence pour des BPR suffisamment fibrés, à la différence des bétons ordinaires, l'apparition systématique de plusieurs zones de localisation de la déformation et le rôle des fibres dans la redistribution des efforts au voisinage d'une zone fissurée.

rhéologie / bétons de poudres réactives / endommagement / traction / localisation de la déformation / mesures cinématiques

\section{Showing of multilocalisation strain phenomena occurring in reactive powders concrete during tensile tests by digital speckle images correlation in lighting white mode}

\begin{abstract}
. digital speckle images correlation, the behaviour in tension of a reactive powders concrete is studied. The measures show several localisation areas of the deformation fields and the role of the fibres in the transmission of the stresses in the neighbourhood of a damaged area.
\end{abstract}

From kinematic observations by granularity in whiting light mode based on a technique of

rheology / reactive powders concrete / damage / tensile test / localisation of deformation /kinematic measures

\section{Introduction}

L'emploi de Bétons Fibrés Ultra-Performants (BFUP) tels que les Bétons de Poudres Réactives (BPR) est a priori intéressant pour la réalisation des structures du Génie Civil en raison des caractéristiques mécaniques et du comportement ductile en traction, non négligeable, de ces matériaux. Divers travaux ont été consacrés à l'étude du comportement macroscopique global en traction d'éprouvettes en BFUP (on peut 
citer par exemple [1,2]). L'originalité de la présente étude réside en une analyse locale du comportement en traction d'un BPR à $2 \%$ de fibres, grâce à des mesures de champs cinématiques. Ces mesures sont obtenues par une méthode optique de granularité en lumière blanche [3], fondée sur une technique d'intercorrélation d'images.

\section{Moyen d'investigation et méthode}

Le dispositif expérimental comporte une machine d'essais mécaniques couplée à une caméra vidéo numérique. Cette dernière acquiert, au cours de la sollicitation, des images de la zone utile de l'éprouvette.

Un point matériel $M$ de la surface est identifié par la répartition de l'intensité lumineuse dans son voisinage. Entre deux images, les composantes du déplacement dans le plan du point $M$ sont calculées par un algorithme d'intercorrélation directe [3]. La dérivation des champs de déplacement permet d'accéder aux autres grandeurs cinématiques telles que vitesse, accélération, déformation, vitesse de déformation. Pour appliquer aux champs de déplacement bruités les opérateurs différentiels correspondants, une technique de lissage local est adoptée : les fonctions d'approximation utilisées sont linéaires par rapport aux variables d'espace, et quadratiques par rapport au temps.

\section{Localisation de la déformation sur un BPR sollicité en traction}

Les éprouvettes en BPR testées sont de forme haltère dont les dimensions sont données figure la. La liaison de ces éprouvettes à la machine d'essais se fait par l'intermédiaire du montage décrit figure $1 b$. Les efforts de traction sont transmis à l'éprouvette par l'intermédiaire de la pièce (3), des plaques latérales (1), et du joint de colle (2) (en rouge sur la figure $1 b$ ).

Les essais présentés sont pilotés par le déplacement de la traverse, avec une vitesse imposée de $10^{-2} \mathrm{~mm} \cdot \mathrm{s}^{-1}$. La sollicitation consiste en trois cycles de charge-décharge en traction, d'amplitude en déplacement croissante.

Pour retranscrire l'intégralité du déroulement de l'essai sur une seule figure, on représente, grâce à une échelle de couleur, l'évolution de la composante axiale $\varepsilon_{11}$ (figure $2 a$ ) du tenseur des déformations linéarisées pour les points situés sur l'axe médian d'une face de l'éprouvette (figure $2 b$ ). Sur la figure $2 b$, on porte, en abscisse, le temps et, en ordonnée, les points de la génératrice en lesquels sont calculées les déformations. L'évolution de la charge au cours du temps est tracée en surimpression (courbe blanche), afin de relier l'évolution des déformations à l'état de chargement de l'éprouvette.

Lors du premier cycle de chargement, le comportement du BPR présente deux phases successives. La première (sur la figure $2 b$ jusqu'à 45 secondes) correspond au comportement élastique du matériau avec des déformations homogènes faibles. La seconde correspond à l'émergence d'hétérogénéités dans le champ de déformation, qui se traduit par l'apparition de plusieurs zones de localisation des déformations

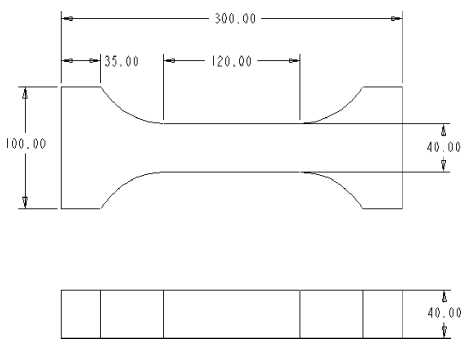

(a)

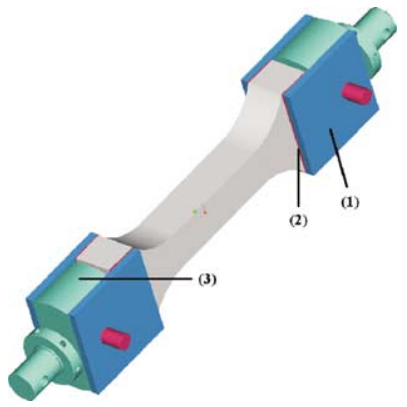

(b)
Figure 1. (a) Géométrie des éprouvettes. (b) Dispositif expérimental de traction.

Figure 1. (a) Sample geometry. (b) Experimental set-up of traction. 


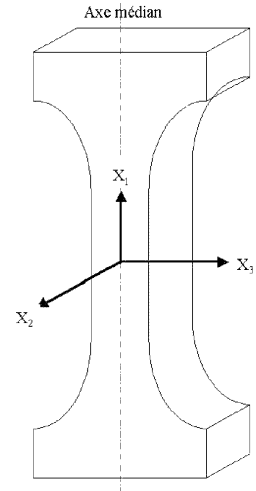

(a)

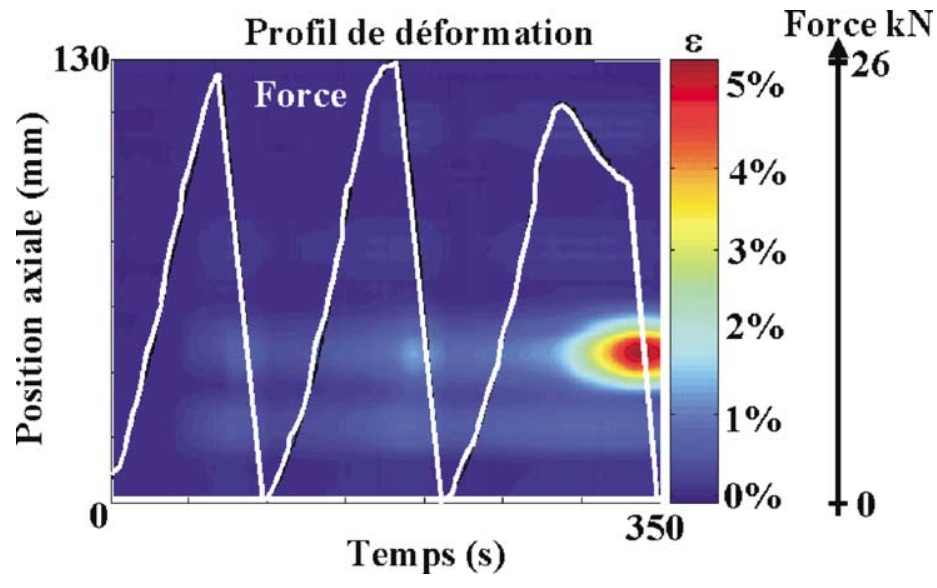

(b)

Figure 2. Évolution au cours du temps du profil des déformations longitudinales sur l'axe médian de l'éprouvette. En surimpression (courbe blanche), évolution de la charge au cours du temps $\left(F_{\max }=26 \mathrm{kN}\right)$.

Figure 2. Time evolution of the lengthwise profiles of the longitudinal component of the strain tensor. Superimposed (white curve): load evolution.

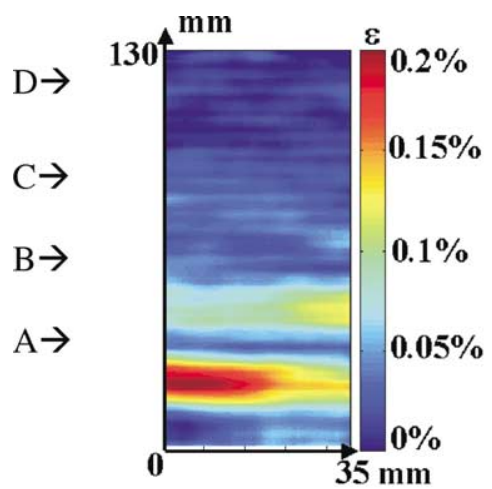

a

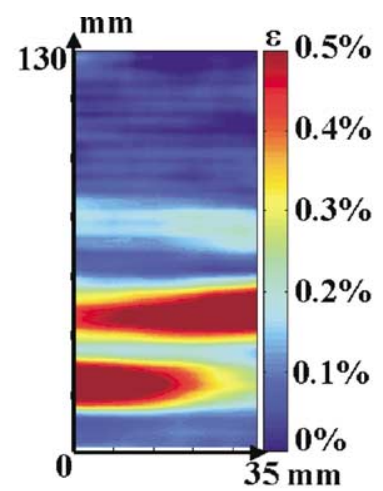

b

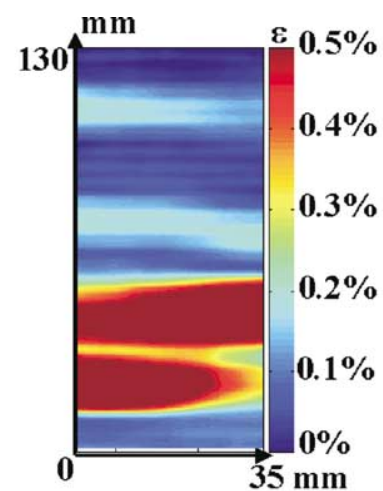

c

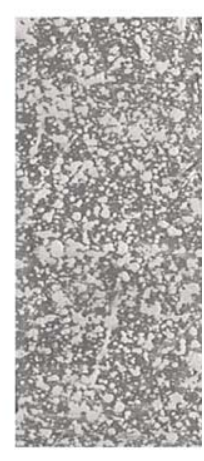

d

Figure 3. (a) Champ de déformation longitudinale à 45 s. (b) Image de l'éprouvette au même instant. (c) Champ de déformation longitudinale à $75 \mathrm{~s}$. (d) Champ de déformation longitudinal à $180 \mathrm{~s}$.

Figure 3. (a) Longitudinal strain field at $45 \mathrm{~s}$. (b) Sample picture at the same time. (c) Longitudinal strain field at $75 \mathrm{~s}$. (d) Longitudinal strain field at $180 \mathrm{~s}$.

(«multilocalisation»). La figure $3 a$, représentant le champ de déformation longitudinal à 45 secondes, illustre ce fait.

On observe, figure $3 a$, deux zones de localisation des déformations alors qu'aucune fissure (figure $3 d$ ) n'est encore visible. A cet instant, il semble que, s'il devait y avoir apparition d'une macrofissure, celleci aurait lieu dans la zone de localisation A (figure $3 a$ ). Or seule la zone B est le lieu de création d'une macrofissure (figure 2).

Si l'on analyse les décharges, on observe (figure 2) des déformations résiduelles plus ou moins importantes suivant l'intensité maximale des déformations atteintes en charge. Les zones A et B sont le siège de déformations résiduelles en fin de décharge, ce qui n'est pas le cas pour la zone $\mathrm{C}$ apparue en 
fin de charge du premier cycle. En deçà d'un certain endommagement, les déformations sont quasiment réversibles.

Le second cycle de chargement met en évidence le caractère «irréversible» de ces zones de localisation, quel que soit l'état de dommage. Dès le début du chargement, on observe une intensification des déformations dans les zones déjà identifiées, mais aussi l'apparition d'une nouvelle zone endommagée D (figure $3 c$ ).

Ces faits expérimentaux ont été systématiquement observés lors de tous les essais de traction directe réalisés sur les BPR. Des observations similaires ont été faites dans le cas d'une poutrelle en BPR sollicitée en flexion 4-points dans la zone en traction. Dans le cas d'un BPR à $3 \%$ de fibres, sollicité en traction, la présence de plusieurs macrofissures peut parfois être observée. Enfin des essais réalisés sur un BPR sans fibres ont montré un comportement en traction qualitativement identique à un béton classique.

De ces essais, il est possible de tirer, sur le comportement en traction d'un BPR suffisamment fibré, les conclusions suivantes :

- la sollicitation imposée conduit systématiquement à l'apparition de plusieurs zones de localisation pour la déformation;

- une ou plusieurs de ces zones conduisent à la création d'une macrofissure, celles-ci ne correspondant pas nécessairement à la première zone de localisation apparue;

- lors de cycles de traction d'amplitude croissante, les zones de localisation ont tendance à se multiplier;

- à la décharge, on observe des déformations résiduelles plus ou moins importantes suivant l'état de dommage associé aux zones de localisation.

L'ensemble de ces phénomènes peut être attribué à la présence des fibres qui, par leur action à distance, favorisent la cohésion du matériau en redistribuant et répartissant les contraintes.

\section{Références bibliographiques}

[1] Rossi P., Renwez S., Guerrier F., Les bétons fibrés à ultra hautes performances - L'expérience actuelle du LCPC, Bull. des Laboratoires des Ponts et Chaussées 204 (juillet-août 1996) 87-95.

[2] Behloul M., Bernier G., Cheyrezy M., Tensile behavior of reactive powder concrete, in: 4th Int. Symp. on Utilization of High-Strength/High-Performance Concrete, Paris, 1996.

[3] Wattrisse B. et al., Analysis of strain localization during tensile tests by digital image correlation, Experiment. Mech. 41 (1) (2001) 29-39.

[4] Richard P. et al., Les Bétons de Poudres Réactives (BPR) à ultra haute résistance (200 à 800 MPa), Ann. l'ITBTP, Série : béton 320 (532) (mars-avril 1995). 